\title{
Targeting Oxidative Stress: A Novel Approach in Mitigating Cancer
}

Pritam Sadhukhan, Sukanya Saha and Parames C Sil*

Division of Molecular Medicine, Bose Institute, Kolkata, India

\begin{abstract}
Reactive Oxygen Species (ROS) plays a vital role in normal cellular homeostasis and development of pathophysiological conditions. Researches round the globe suggest that cancer cell possesses higher amount of intracellular ROS and impaired metabolic activity along with mitochondrial dysfunction. ROS can initiate cancer, but the primary endogenous elevation of oxidants in malignant tissues leave them more susceptible to secondary stresses. Despite the crucial role of ROS in the development of cancer, anticancer therapies are suggested both by scavenging the excessive intracellular ROS and also by inducing ROS generation through exogenous oxidative insult. In this commentary, we have discussed the dual effect of ROS on the development cancer and emergence of novel anti-cancer therapies by modulating the accumulation of ROS.
\end{abstract}

Keywords: Reactive oxygen species; Oncogenes; Tumor suppressor genes; antioxidants

\section{Commentary}

Oxidative stress defines an impaired physiological state where intracellular Reactive Oxygen Species (ROS) increases within the body $[1,2]$. It is essentially an imbalance between the production of free radicals and the ability of the body to counteract or detoxify their harmful effects through neutralization by endogenous antioxidants [3]. The severity of oxidative stress is directly proportional to the amount of ROS generated. ROS are chemically reactive molecules containing oxygen. Moderate amount of ROS present within the cells facilitates cell proliferation and maintains their normal homeostasis, but at higher concentrations it can impair different cellular functions and ultimately in many scenarios, leads to the development of malignancy or cell death $[4,5]$. There is an equilibrium between a free radical/ROS formation and endogenous antioxidant defence mechanisms, but if this balance is perturbed, it can produce oxidative stress phenomenon [6]. The modulation of oxidative stress is a determining factor in both the development of tumour and target of anticancer therapies. The molecular signalling pathways associated with tumorigenesis also interferes with the intracellular ROS metabolism. Cancer cells have impaired ROS metabolism and it exhibits a higher level of intracellular ROS than normal somatic cells [7]. There is a controversy in the research community about the exact nature and impact of oxidative stress on the development of cancer and its beneficial effects employed on different anti-cancer therapies [8].

Nearly 35 years back, in 1981, it was first reported that intracellular ROS metabolism is directly linked with the cellular transformation [9]. It is also a fact that cancer results due to the activation of several oncogenes and simultaneous inactivation of the tumor suppressor genes. At a moderate level, ROS can activate several molecular pathways, which induces tumor development. For instance, ROS can stimulate the activation and phosphorylation of several kinases like, mitogen-activated protein kinase (MAPK), extracellular signalregulated kinase (ERK), JUN N-terminal kinase (JNK) and induce the expression of cyclin D1, which is known to facilitate cell cycle progression and cell proliferation [10]. Supporting the fact that ROS facilitates tumorigenesis, it is observed that ROS can potentially impair intracellular antioxidant defence and ROS scavenging machinery by suppressing the phosphatase and tensin homolog (PTEN) and protein tyrosine phosphatases (PTPs) [11].

As discussed here, though the moderate level of intracellular ROS promotes cellular proliferation, a high concentration of ROS can cause severe cellular damage and even cell death. So the cancer cells develop a very strong antioxidant capacity to maintain cellular homeostasis. Despite the presence of a significantly powerful antioxidant system, it is observed that cancer cells are more prone to damage due to ROS accumulation than the normal somatic cells [12] (Figure 1).

There are several genes present in our body which express differentially under specific stress conditions. Under oxidative stress, some of the genes in turn regulate the expression various antioxidant genes and affect the intracellular redox environment. The transcription factor, nuclear factor erythroid 2-related factor 2 (NRF2) is identified as one of the most important regulator of several anti-oxidative genes [13]. To be very precise, it mainly regulates the intracellular production of glutathione (GSH) by interfering the expression of glutamate-cysteine ligase (GCL) complex and NADPH-generating enzymes [13]. Other than GSH it is also known to alter the expression of several glutathione S-transferases. Like NRF-2, the tumour suppressor p53 and forkhead box $\mathrm{O}$ (FOXO) family of transcription factors are also known to have

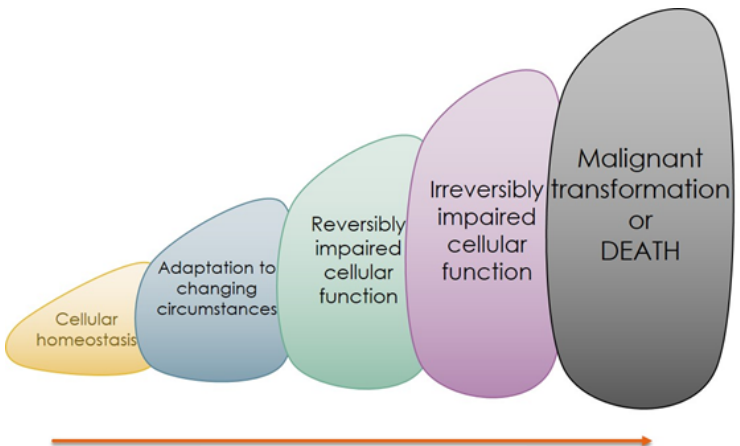

Level of Intracellular ROS

Figure 1: Effect of intracellular ROS level on normal cellular homeostasis.

*Corresponding author: Parames C Sil, Division of Molecular Medicine, Bose Institute, Kolkata, India, Tel: 9133-25693243; Fax: 9133-2355-3886; E-mail: parames@jcbose.ac.in, parames_95@yahoo.co.in

Received: November 30, 2015; Accepted: December 08, 2015; Published December 31,2015

Citation: Sadhukhan P, Saha S, Sil PC (2015) Targeting Oxidative Stress: A Nove Approach in Mitigating Cancer. Biochem Anal Biochem 4: 236. doi:10.4172/2161. 1009.1000236

Copyright: (c) 2015 Sadhukhan P, et al. This is an open-access article distributed under the terms of the Creative Commons Attribution License, which permits unrestricted use, distribution, and reproduction in any medium, provided the original author and source are credited. 
significant anti-oxidative property by regulating different individual as well as overlapping antioxidant genes. Both of these family of genes promote the stabilization of NRF2 [14].

The oncogenes and tumour suppressor genes, apart from these genes mentioned so far, which regulate the expression of different antioxidant genes, has a substantial impact on tumorigenesis initiation, its development and progression. Unlike NRF-2 and FOXO, interestingly p53 is known to stimulate both pro- and anti-oxidant genes. The role of different tumour suppressor genes is more significant than the oncogenes in terms of regulation of oxidative stress. As mentioned earlier the tumour suppressor genes get suppressed during the development of cancer, but the suppression of several tumour suppressor genes results in upregulation or downregulation of various antioxidant genes [15].

So far we have discussed about the controversial dual nature of ROS; in one hand it can develop tumor and can suppress tumor formation on the other. For this behavioural characteristic of ROS two kinds of therapeutic approach have emerged, first by scavenging the elevated amount of intracellular ROS and the other by inducing intracellular ROS formation. In case where intracellular ROS induces mutagenesis and results in cell proliferation, exposure to various antioxidants like Vitamin E, selenium, various polyphenols etc. [16] have been found to be effective. On the other hand exogenous ROS insult, which elevates the intracellular ROS level, is found to be very useful in selective killing of cancer cells barring the normal cells.

There are several instances where antioxidant supplementation results in reduction of cancer formation in gastric and hepatic cancer patients and increased cancer mortality in case of lung cancer patients [17]. At the same time, it is also reported that use of antioxidants as a therapeutic option for cancer, is limited to some genetic variants and highly metastatic carcinomas [18]. Several in vitro and animal experiments proved that dietary intake of different antioxidant rich foods (foods enriched in genistein, curcumin, etc.) are beneficial against cancer $[16,19]$. On this regard several genetically engineered food, designed to contain higher level of antioxidants, has gained much importance in combating this deadly disease.

Cancer cells resist elevated ROS induced damage and death by increasing their antioxidant defences. This indicates that therapies which induces intracellular ROS level and impair the antioxidant defence mechanism of the cancer cells may have better effectivity in terms of minimum side effect and drug resistance.

Finally, it must be underlined that ROS plays a critical role in the initiation, progression and metastasis of cancer. At the same time, accumulation of intracellular ROS can again lead to cell death. So, cancer can be prevented through regulating the level of ROS either by scavenging the intracellular ROS or by their accumulation via exogenous oxidative insult. So, molecules targeting ROS could be the upcoming therapy for cancer and anticancer drug discovery studies in future.

\section{References}

1. Rossigno DA, Frye RE (2012) A review of research trends in physiological abnormalities in autism spectrum disorders: immune dysregulation, inflammation, oxidative stress, mitochondrial dysfunction and environmental toxicant exposures. Mol Psychiatry 17: 389-401.

2. Sinha K, Das J, Pal PB, Sil PC (2013) Oxidative stress: the mitochondriadependent and mitochondria-independent pathways of apoptosis. Arch Toxicol 87: $1157-1180$
3. Rashid K, Sinha K, Sil PC (2013) An update on oxidative stress-mediated organ pathophysiology. Food Chem Toxicol 62: 584-600.

4. Morgan MJ, Liu ZG (2011) Crosstalk of reactive oxygen species and NF-îمB signaling. Cell Res 21: 103-115.

5. Ogrunc M, Di Micco R, Liontos M, Bombardelli L, Mione M, et al. (2014) Oncogene-induced reactive oxygen species fuel hyperproliferation and DNA damage response activation. Cell Death Differ 21: 998-1012.

6. Farooqi AA, Li KT, Fayyaz S, Chang YT, Ismail M, et al. (2015) Anticance drugs for the modulation of endoplasmic reticulum stress and oxidative stress. Tumour Biol 36: 5743-5752.

7. Poillet-Perez L, Despouy G, Delage-Mourroux R, Boyer-Guittaut M (2015) Interplay between ROS and autophagy in cancer cells, from tumor initiation to cancer therapy. Redox Biol 4: 184-192.

8. Pizzimenti S, Toaldo C, Pettazzoni P, Dianzani MU, Barrera G (2010) The "twofaced" effects of reactive oxygen species and the lipid peroxidation product 4-hydroxynonenal in the hallmarks of cancer. Cancers (Basel) 2: 338-363.

9. Oberley LW (1988) Free radicals and diabetes. Free Radic Biol Med 5: 113-124

10. Ranjan P, Anathy V, Burch PM, Weirather K, Lambeth JD, et al. (2006) Redoxdependent expression of cyclin $\mathrm{D}$ and cell proliferation by Nox1 in mouse lung epithelial cells. Antioxid Redox Signal 8: 1447-1459.

11. Leslie NR, Bennett D, Lindsay YE, Stewart H, Gray A, et al. (2003) Redox regulation of PI 3-kinase signalling via inactivation of PTEN. EMBO J 22: 55015510.

12. Diehn M, Cho RW, Lobo NA, Kalisky T, Dorie MJ, et al. (2009) Association of reactive oxygen species levels and radioresistance in cancer stem cells. Nature 458: 780-783.

13. Sporn MB, Liby KT (2012) NRF2 and cancer: the good, the bad and the importance of context. Nat Rev Cancer 12: 564-571.

14. Cairns RA, Harris IS, Mak TW (2011) Regulation of cancer cell metabolism. Nat Rev Cancer 11: 85-95.

15. Kruiswijk F, Labuschagne CF, Vousden KH (2015) p53 in survival, death and metabolic health: a lifeguard with a licence to kill. Nat Rev Mol Cell Biol 16 393-405.

16. Saha S, Sadhukhan P, Sil PC (2014) Genistein: A Phytoestrogen with Multifaceted Therapeutic Properties. Mini Rev Med Chem .

17. Blot WJ, Li JY, Taylor PR, Guo W, Dawsey S, et al. (1993) Nutrition intervention trials in Linxian, China: supplementation with specific vitamin/minera combinations, cancer incidence, and disease-specific mortality in the general population. J Natl Cancer Inst 85: 1483-1492.

18. Hurst R, Hooper L, Norat T, Lau R, Aune D, et al. (2012) Selenium and prostate cancer: systematic review and meta-analysis. Am J Clin Nutr 96: 111-122.

19. Ghosh S, Banerjee S, Sil PC (2015) The beneficial role of curcumin on inflammation, diabetes and neurodegenerative disease: A recent update. Food Chem Toxicol 83: 111-124. 